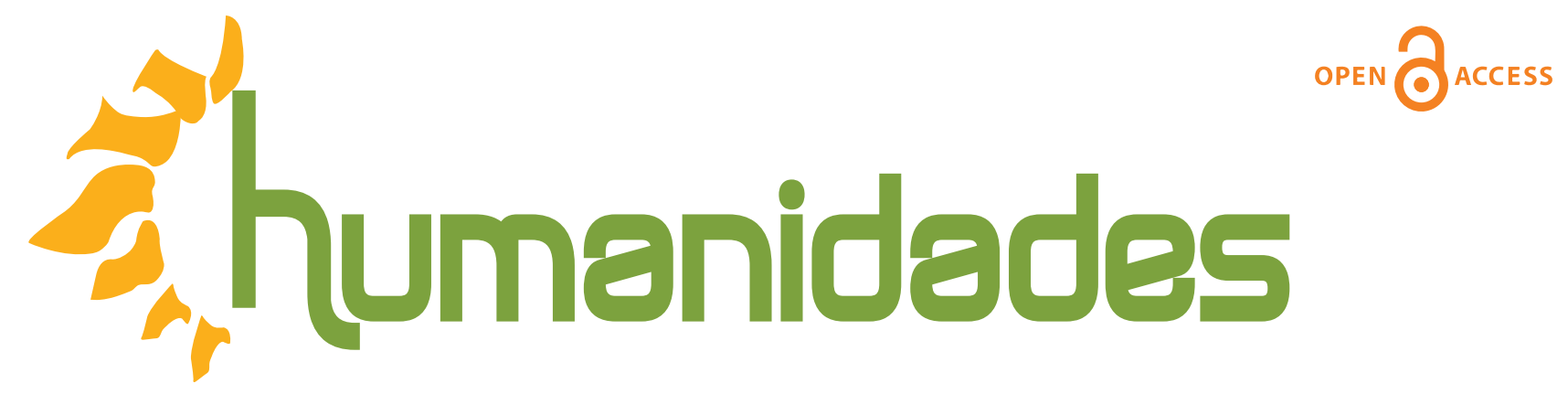

Revista de la Escuela de Estudios Generales, Universidad de Costa Rica

Julio-diciembre, 2019 •Volumen 9, número 2 • EISSN 2215-3934・pp. 1-17

Recibido: 07-Enero-2019 Aceptado: 29-Enero-2019

\title{
Diseño de la ciudad, normalización y vida social
}

DOI: https://doi.org/10.15517/h.v9i2.37267

\section{Elizabeth Fernández Rojas}

Doctora en Humanidades

Universidad Autónoma del Estado de México, México

Correo electrónico: efernandezr705@profesor.uaemex.mx / eliferoj@hotmail.com

Todos los derechos reservados. Universidad de Costa Rica. Esta revista se encuentra licencida con Creative Commons. Reconocimiento-NoComercial-SinObraDerivada 3.0 Costa Rica. Correo electrónico: humanidades@ucr.ac.cr/ Sitio web: http: //revistas.ucr.ac.cr/index.php/humanidades 


\section{Diseño de la ciudad, normalización y vida social}

\section{Resumen}

En tiempos actuales de globalización como los de nuestra competencia, la

Palabras clave: idea de constituir una ciudad desde la institución de su diseño como eje rector esencial, resulta una determinación relevante tanto para la gestación del espacio físico adaptado en cuestión, como para el desarrollo cotidiano de la humanidad; pues se contempla que en la medida en que exista una constitución integral regida por la planeación adecuada de sus componentes espaciales y temporales, la problemática urbana que aqueja a las grandes ciudades con toda probabilidad disminuirá. Ante tal distinción resulta importante cuestionar ¿quién es la instancia encargada de diseñar o rediseñar la ciudad?, ¿el Estado, los especialistas, la sociedad? Para nosotros resulta evidente que la respuesta a tales interrogantes, en gran medida, se encuentra implícita en la efectividad y aplicación conducente de los mecanismos normativos que presumen favorecer la continuidad prospectiva de todo espacio físico adaptado, situación particular que, en teoría, debe facilitarle a las personas la interacción, no solo con otras personas, sino también en reciprocidad, con el espacio físico que lo rodea en la ejecución de su vida diaria y las exigencias que el entorno social le demanda. 


\title{
City Design, Normalization, and Social Life
}

\begin{abstract}
In current times of globalization like those of our competition, the idea of constituting a city from the institution of its design as an essential guiding axis, is a relevant determination both for the gestation of the adapted physical space in question, as well as for the daily development of the humanity; because it is contemplated that insofar as there is an integral constitution governed by the adequate planning of its spatial and temporal components, the urban problems that afflict to big cities will in all probability decrease. In front such distinction, it is important to question: Who is the entity in charge of designing or redesigning the city? State, specialist, society? For us it is evident that the answer to such questions, in a large extent, is implicit in the effectiveness and conducive application of the normative mechanisms that presume to favor the prospective continuity of any adapted physical space, a particular situation that in theory, should facilitate the person the interaction not only with other person, but also in reciprocity, with the physical space that surrounds them in the execution of his daily life and the demands that the social environment demands.
\end{abstract}

Keywords: 


\section{Diseño de la ciudad, normalización y vida social}

¿Qué se entiende por constitución de la ciudad? Para el desarrollo del tema concerniente es importante destacar que constituir el espacio físico adaptado, mejor conocido como ciudad, para nosotros adquiere una connotación que trasciende la idea adjetiva de representación de una parte del todo que simboliza el contexto urbano, de ahí se contempla que dimensionar únicamente la constitución del sujeto que la dinamiza, involucra anticipadamente la reducción de la vivencia urbana. Constituir la ciudad implica, además, la exposición sistémica y sistemática de sus configuraciones por conducto de los elementos ordenados en tanto normas y procedimientos que retribuyen la totalidad de la recreación urbana y social.

Bajo este esquema, contemplamos que un espacio físico adaptado que soslaya la prefiguración de su estructura, deja de lado las particularidades que en teoría deberían facilitar también la constitución del ser para su adecuada vivencia en sociedad. Pues, omitir dicha solicitud ontológica, precipita, por una parte, la decadencia de los procesos cognitivos que ejercitan las preguntas que cuestionan por la propia ciudad; nos referimos a la epistemología que las instituye. Por otra parte, demeritan la técnica que gestiona la constitución y distribución espacial de su equipamiento e infraestructura.

Como parte de los indicadores o constituyentes que motivan el desarrollo del ser en función del diseño de la ciudad, encontramos por señalar algunos ejemplos: incremento de parques, zonas arboladas, zonas comerciales, generación de espacios recreativos, etc. Tales indicadores permitirán de manera conjunta el desarrollo, tanto de la ciudad como la de aquellos que la habitan, al tiempo que interactúan en sinergia con la naturaleza de cada lugar.

Para conceder continuidad al desarrollo del tema en competencia, es de suma importancia responder a la interrogante ¿qué se entiende por planeación o diseño adecuado de la ciudad? Todo espacio físico adaptado que se constituye por vez primera demanda la elaboración anticipada de los diseños, planes, estrategias y programas que formalizan la culminación de las metas a alcanzar previas a su puesta en marcha. 
Diseño de la ciudad, normalización y vida social

Para el caso que aquí compete, en tanto que las ciudades actuales se encuentran configuradas, se contempla que es primordial prever los instrumentos jurídicos que normalicen, en materia de ordenamiento territorial, las estrategias que orienten el rediseño y el desarrollo espacial y temporal de la ciudad a través del diálogo entre aquellos agentes o elementos que la integran.

Una normalización anacrónica de la ciudad es inútil, pues desvirtúa el telos de toda ciudad, generando problemáticas urbanas que aquejan en mayor proporción a las grandes ciudades actuales ${ }^{1}$, como lo son para el caso de la República Mexicana, la Ciudad de México, el Estado de México y su capital Toluca, Guadalajara y Monterrey, entre otras, de no menor relevancia. Por citar un ejemplo sucinto de lo que se habla, es posible referir la normalización prevalente en cuanto al uso de suelo en sus variables de densidad, altura o coeficientes de aprovechamiento, para la Zona Metropolitana del Valle de Toluca (ZMVT), en tanto que, el propio Plan Municipal de Desarrollo Urbano (PMDU) Vigente 2005-2014 reconoce de forma sutil las limitaciones normativas que históricamente han demeritado, y lo siguen haciendo, la productividad urbana eficaz y eficiente de la Zona Metropolitana en referencia:

En el ámbito de las áreas urbanas, se puede concluir que el PMDU, si bien ha sido de utilidad en la operación de la normatividad contenida en este instrumento de regulación, ya que ha posibilitado la redensificación y la ocupación de los vacíos urbanos, en aprovechamiento de las áreas servidas con infraestructura, servicios y equipamiento urbanos; también se detectó que adolece de ajustes particulares en la normatividad por sectores urbanos; y se concretizan en los siguientes puntos:

-Existen áreas que, en el PMDU de 2005, tenían asignada la clasificación de áreas urbanas o urbanizables y que, en el vigente de 2014, se le cambió por el de áreas no urbanizables, tal es el caso de superficies ubicadas en la localidad, Sebastián Lerdo de tejada, que habría que devolver el uso en como un derecho creado de los propietarios de estos predios. 
-La normatividad de algunas otras zonas en cuanto a densidad y superficie y frente mínimo de los lotes para subdivisión y edificación, no responden a la situación real del tamaño de lotes existentes en las áreas urbanas, lo que impide la regularización de las construcciones.

-Que, con la normatividad del PMDU vigente no es posible promover la redensificación urbana; ni el crecimiento vertical, por lo que no son autorizadas solicitudes de cambio de densidad o de altura.

-Que al momento no es permisible la mezcla de usos en la mayor parte de las zonas urbanas. (pp. 15-16)

Adicionalmente al uso del suelo normalizado se le suma la enorme y continua migración de gente que va del campo a la ciudad. De ahí que la problemática derivada de normalizaciones anacrónicas se acreciente, afectando desproporcionadamente la morfología de tales ciudades, puesto que la migración como tal, responde a una diversidad de factores que a su vez incorporan los órdenes económicos, políticos, educativos, culturales, religiosos y sociales, por señalar algunos de relevancia, trayendo consigo afectaciones y desregulaciones urbanas de seriedad que modifican considerablemente los componentes que le constituyen, entre los que se encuentran: insuficiencia y deterioro de infraestructura y equipamiento urbano, escasez y encarecimiento de la vivienda, escasez de fuentes de trabajo, delincuencia, contaminación ambiental, entre otros.

¿Quién es la instancia encargada de diseñar o rediseñar la ciudad: el Estado, los especialistas o la sociedad? En la intención de trascender cualquier formalidad de la retórica ambigua, se contempla que el consenso efectivo mediante el diálogo veraz y la aplicación efectiva de la norma, formalizan la interacción tripartita entre los entes denominados con anterioridad. No se trata de que una u otra parte se posicione unívoca y jerárquicamente en la intención de regir los preceptos de la planeación y diseño en torno a las ciudades modernas, sino que se requieren visiones que conjunten las diferentes versiones y que se haga lecturas de las ciudades en torno a la contemplación de sus necesidades para la vida empática en sociedad. 
En el interés de caracterizar una visión especifica de la constitución de la ciudad y ofrecer perspectivas de respuesta, se entiende que al inicio el hombre se encuentra solo e inicia su vida sin establecerse en un lugar y espacio determinado, es decir, es un nómada; sin embargo, a través del tiempo comienza a surgir la necesidad de implementar, de crear y diseñar un espacio en el que pueda establecerse con el propósito de convivir, desarrollarse e interactuar con su entorno tanto natural, como artificial y social. Ya lo señala Rousseau (2011):

(...) todo empieza a cambiar de aspecto. Los hombres errantes hasta entonces por los bosques, tienen ahora un asentamiento más fijo, y poco a poco se acercan, se reúnen en agrupaciones diversas, formando por último en cada territorio una nación particular, unida en costumbres y caracteres, no mediante leyes y reglamentos (p. 175)

De lo anterior, se deduce principalmente que el diseño de la ciudad le corresponde al ser humano en interacción con la sociedad, en donde no existe regulación alguna respecto al diseño de la ciudad; más bien, se toma en consideración los aspectos que permitan cubrir las necesidades básicas de cercanía y protección de los individuos.

Con tal perspectiva de concentración humana ocurre la génesis de la comunidad, y consecuentemente, la denominada civitas o polis, en la cual, propiamente no existía regulación específica y mucho menos técnica para el diseño y constitución de la ciudad como actualmente se le conoce. A ello se le sumaría otro de los factores que influye proporcionalmente en la adjudicación de la categoría de ciudad, mismo que incide en mantener determinado número de habitantes.

De ahí que sea posible destacar que el diseño de la ciudad como primer momento, formalmente, inicia en la constitución de la comunidad con las primeras chozas o espacios diseñados para resguardo de las inclemencias naturales, de manera empírica, sin que existiera como tal una regulación para el diseño propiamente de la ciudad. Con el fortalecimiento de las determinaciones políticas, de las núbiles instituciones y la creación del Estado, empiezan a estandarizarse los mecanismos regulatorios que permean en todos los aspectos que le conciernen, entre otras dimensiones, a la planeación de los asentamientos humanos y al equipamiento e infraestructura que ello conlleva. 
Para reforzar lo anterior, es menester trasladarse al caso concreto de las principales ciudades de América Latina. Ahí se considera que los inicios de la regulación de la planificación -sin que con ello se pretenda negar la existencia de una planificación de los centros urbanos prehispánicos- se gestan a partir de la conquista de los españoles, precisamente, cuando estos manifiestan en el trazo y diseño de la ciudad la influencia europea de los asentamiento coloniales; posteriormente, con la aplicación de las ordenanzas provenientes del régimen de Felipe II en 1573, que estipulaban que las calles deberían estar dispuestas en damero con una plaza central, en torno a la cual se agrupaba la iglesia y los edificios reales y civiles, traza que en la actualidad aún pervive en nuestras ciudades.

Posteriormente, la planificación de estos centros urbanos seguirá con las Leyes de Indias que dieron forma y organización, aplicando diversos criterios para zonificación, edificación, orientación y accesibilidad, así como para el desarrollo de actividades agrícolas y económicas dentro y fuera de la ciudad (Gutiérrez, 2009, p. 54).

Es así como el diseño y planeación de las ciudades, como tal, comienza a ser regulado por ordenamientos jurídicos que establecen formalidades a las que estas deben circunscribirse; con ello es posible contemplar, a su vez, el inicio de la normalización de los diferentes objetos de diseño que constituyen a la ciudad, por lo que de ahí en adelante la planeación se considerará por cuenta del Estado colonial.

Para el caso mexicano, la normalización de la Planeación Urbana Moderna inicia con la primera Ley General de Asentamientos humanos (LGAH), publicada en el Diario Oficial de la Federación el 26 de mayo de $1976^{2}$, la cual establecía lo siguiente:

ARTÍCULO 1.- Las disposiciones de esta Ley son de orden público e interés social y tienen por objeto: 
I.- Establecer la concurrencia de los Municipios, de las Entidades Federativas y de la Federación para la ordenación y regulación de los asentamientos humanos en el territorio nacional; II.- Fijar las normas básicas para planear la fundación, conservación, mejoramiento y crecimiento de los centros de población; y III.Definir los principios conforme a los cuales el Estado ejercerá sus atribuciones para determinar las correspondientes provisiones, usos, reservas y destinos de áreas y predios.

Con el establecimiento de la Ley antes mencionada se positiviza el derecho del Estado para establecer las medidas pertinentes en lo concerniente a los asentamientos humanos $^{3}$, con el interés de que tal regulación mejore las condiciones de vida de la población tanto urbana como rural; de ahí que destaquen conforme lo establecido en el Artículo 3 los siguientes ordenamientos:

I.-El aprovechamiento en beneficio social, de los elementos naturales susceptibles de apropiación, para hacer una distribución equitativa de la riqueza pública; II.El desarrollo equilibrado del país, armonizando la interrelación de la ciudad y el campo y distribuyendo equitativamente los beneficios y cargas del proceso del desarrollo urbano; III.-La distribución equilibrada de los centros de población en el Territorio Nacional, integrándolos en el marco del desarrollo nacional; IV.-La adecuada interrelación socio-económica de ciudades en el sistema nacional; V.-La más eficiente interacción entre los sistemas de convivencia y de servicios en cada centro de población, particularmente, la creación y mejoramiento de condiciones favorables para la relación adecuada entre zonas industriales y de vivienda de trabajadores, el transporte entre ambas, y las justas posibilidades de trabajo y descanso; VI.-El fomento de ciudades de dimensiones medias a fin de evitar las que por su desproporción producen impactos económicos negativos y grave deterioro social y humano; VII.-La descongestión de las grandes urbes; VIII.-El mejoramiento de la calidad de la vida en la comunidad; IX.-La mayor participación ciudadana en la solución de los problemas que genera la convivencia en los asentamientos humanos; X.-La regulación del mercado de los terrenos. Además, el de los inmuebles dedicados a la vivienda popular; y XI.-La promoción de obras para que todos los habitantes del país tengan una vivienda digna. 
Asimismo, se establecía que la ordenación y la regulación de los asentamientos humanos se llevarían a través del Plan Nacional de Desarrollo Urbano, los Planes Estatales de Desarrollo Urbano, los Planes Municipales de Desarrollo Urbano y los Planes de Ordenación de las Zonas Conurbadas, promoviendo en todo momento la participación de los distintos grupos sociales que integran la comunidad en la elaboración de dichos planes.

Así, a lo largo de la historia, se gestaron diferentes esfuerzos por parte del Estado para regular lo concerniente a los asentamiento humanos, tal es el caso de la Secretaría de Asentamientos Humanos y Obras Públicas ${ }^{4}$ (SAHOP) cuyo principal propósito consistía en la planeación de los centros de población, de la ecología urbana, del equipamiento, del suelo y la vivienda, siendo en el marco de las atribuciones de esta secretaría que se elabora el primer Plan Nacional de Desarrollo Urbano en 1978, institucionalizando de esta forma la acción del Estado en materia de organización del territorio.

Es claro y evidente que, desde el inicio de la positivización en México respecto a los asentamientos humanos, existe una colaboración entre los diferentes niveles de gobierno y los ciudadanos, en función de las estrategias y planeación que se pretende implementar en el diseño de la ciudad y su contexto natural, situación que en menor o mayor medida permanece vigente por conducto del diálogo.

De tal orden de ideas es posible destacar que, en sus inicios, el diseño de la ciudad, incluidos sus asentamientos humanos, le correspondía en principio a la sociedad, es decir, su única autorregulación era satisfacer sus necesidades, subsecuentemente, con la creación del Estado, le compete a este la totalidad de los ordenamientos y su regulación. De aquí que cobre importancia la afirmación de E. Kant (2010) cuando destaca que: 
Diseño de la ciudad, normalización y vida social

La realización de la idea requiere un esquema, es decir, esencia variedad y orden de las partes, ambas cosas determinadas a priori por el principio según el cual se rige el fin. El esquema que no se traza de acuerdo con una idea, es decir, partiendo del fin primordial de la razón, sino desde un punto de vista empírico, de acuerdo con intenciones que se presentan accidentalmente (cuyo número no puede conocerse de antemano), nos ofrece una unidad técnica; el que surge en cambio, como resultado de una idea (donde la razón propone los fines a priori y no los espera empíricamente) funda una unidad arquitectónica (p. 603)

Partiendo del supuesto de que al Estado es a quien le compete el diseño de la ciudad en conjunto con la sociedad, entonces, ¿por qué la problemática urbana va en aumento? Habría que analizar si es un diálogo el que se pretende establecer entre los involucrados en el diseño. Ya lo señala Agustín Domingo Moratalla citado por Esquivel (2012) cuando afirma: "no es posible entendernos bajo el régimen del sometimiento o del domino del otro, sino en la dirección de un encuentro dialogal y vivencial" (pp. 38-39).

De ahí que se contemple que el camino adecuado es el diálogo con posibilidad de comprensión y de conversación veraz con el otro, puesto que ello se convierte en un diálogo interminable (Esquivel, 2012, p. 30) y lo que es patente en la interacción entre Estado y sociedad es que ni diálogo ni veracidad se dan, mucho menos con los especialistas del diseño, aun cuando así la ley lo establece en su normalización.

Con ello se evidencia que el rediseño de las ciudades actuales ha sido poco o escasamente planeado por las instancias encargadas, tanto como por parte de ciertos particulares, quienes su prioridad inmediata radica en satisfacer necesidades básicas o comerciales sin prever posibles riesgos de impacto a la protección civil, a la planeación urbana, a la ambiental, o a la seguridad familiar, entre muchos otros de relevancia, propiciando con ello el inadecuado diseño de la ciudad, lo cual ocurre, quizás, por el hecho de evadir los requisitos que para tales efectos prevé la legislación vigente, o bien, por desconocimiento de tal regulación. 
Por su contraparte, las instancias encargadas de vigilar el cumplimiento de la regulación establecida soslayan su competencia permitiendo la vertiginosa irregularidad de los asentamientos humanos y, con ello, fomentan la aparición de riesgos elevados por usos y destinos de suelo indeterminados, así como la desaceleración del diseño de la ciudad en función de su propio detrimento.

En función del rediseño adecuado de la ciudad, más allá de otros intereses, se requieren acuerdos veraces entre las partes, mediados por argumentos racionales que faciliten el consenso real y, por consiguiente, la plenitud de una acción social en aras del bien común. Ya lo plantea con claridad Habermas en función de las pretensiones de validez o actos del habla: "inteligibilidad, veracidad, rectitud normativa y verdad" (Cfr. Esquivel, 2016, pp. 126-142), considerados como indispensables para la integración de toda comunidad de diálogo, útiles para la coordinación de las actividades humanas; para el caso, en torno a la realización del Plan de Desarrollo Urbano y lo concerniente al diseño de la ciudad.

Se entiende por la primera pretensión de validez lo relativo a la claridad del propio discurso, de aquello de lo que se dice se requiere un mínimo de entendimiento entre los dialogantes, pues de lo contrario el discurso no cumple con la primera de las pretensiones, es decir, se vuelve ininteligible, generando la imposibilidad de entender, o bien, tergiversar lo que se pretende decir. En la primera pretensión, la inteligibilidad, se requiere de la disposición intersubjetiva de ser interpelados tanto por parte de la sociedad como por del Estado con la finalidad de dar el seguimiento adecuado respecto al rediseño de la ciudad, de tal forma que permita llegar a un consenso que otorgue respuestas a las preguntas a las que se ha aludido.

En lo que respecta a la segunda pretensión: veracidad, se considera que es la actitud originaria de credibilidad, e incluso, podrá entenderse como la buena fe del discurso entre los interlocutores, en el que debe existir congruencia entre lo que se habla y las acciones; se puede decir que se establece la confianza y sinceridad respecto a los pensamientos de lo que se dice y de lo que se proyecta en las acciones. Si cualquiera de las partes no es sincera en relación con lo que piensa, habla y hace, difícilmente puede existir la veracidad, pues no está sujeta a la comprobación empírica. 
Diseño de la ciudad, normalización y vida social

En este supuesto cabe hacer mención en lo concerniente al gobierno y a los ciudadanos con respecto a las estrategias de planeación y rediseño que se pretendan implementar en torno a la ciudad y a su contexto natural; habría que cuestionar si existe veracidad en sus interacciones, pues el momento en el que se puede cuestionar tal pretensión de validez estriba en la acción, donde con facilidad se puede establecer el fingimiento de las intenciones, que solo se limitan a tal cosa, intención por parte de uno de los dialogantes, (que generalmente suele ser la parte que representa los intereses del Estado) lo cual genera una comunicación distorsionada o ficticia.

La tercera pretensión: rectitud normativa, tiene que ver con la relación intersubjetiva en el campo de la comunicación e implica la aceptación de normas que rigen la relación del comportamiento entre hablante y oyente, es decir, se deben tomar en consideración los intereses de todos los implicados o afectados por tales normas con la finalidad de alcanzar consensos. Cuando el hablante (que en este supuesto sería el Estado) decreta un mandato, el acuerdo va a depender de que los participantes (sociedad e incluso los especialistas en el diseño) consideren normativamente correcta la acción, respecto a las normas planteadas, mismas que pueden ser aceptadas o rechazadas, es decir, es posible cuestionar las normas.

Por último, la pretensión: verdad, tiene que ver con los contenidos, consiste en la posibilidad de alcanzar un consenso racional sobre lo que se dice; abre la posibilidad a la discusión para defender o rechazar los contenidos con afirmaciones o explicaciones, por lo tanto, una vez cumplidas las pretensiones de validez se puede llegar a la verdad consensuada racionalmente y, en consecuencia, el diálogo se cumpliría. Para el caso que nos concierne, bajo los supuestos establecidos como pretensiones de validez o actos del habla, sería importante cuestionar ¿el diálogo entre los involucrados en el rediseño de la ciudad se da de manera real o simulada?, la respuesta a tal interrogante queda patente en la problemática que en lo nacional, estatal y local aqueja a las diferentes ciudades. 
Entre las acciones que se detectan respecto a la implementación necesaria de todo diálogo en torno al rediseño de la ciudad, es posible afirmar que el diseño por conducto de los actores implicados omite incorporar aspectos naturales inherentes a la ciudad que faciliten el desarrollo del ser humano, no obstante, si los incorpora, los mismos son enfocados al desarrollo económico de cierta élite, y generalmente, sobre la idea de una creciente gentrificación; mientras que el ciudadano promedio pervive en la periferia ignorado por el Estado y agobiado por la regulación establecida que le obliga, por necesidad o desconocimiento, como ya ha sido mencionado, a ignorar su cumplimiento.

Ya ha sido mencionado también que en las ciudades modernas la regulación del rediseño de la ciudad le compete al Estado, pues esta entidad es la encargada de establecer las regulaciones respecto a lo que se puede o no hacer en cuanto al cumplimiento urbano de la ciudad, independientemente, de si se trata de la iniciativa y los bienes privados o públicos, las posibilidades de rediseño urbano se encuentran supeditadas al cumplimiento de normalizaciones legalmente decretadas, muchas de las cuales. generalmente, favorecen al capital en detrimento de particulares y, a la vez, favorecen el avejentamiento de la ciudad. Cosa similar sucede con las urbes rurales y los malogrados y denominados "pueblos mágicos", producto de otra investigación.

Entonces, como se pudo ver, toda vez que es patente el hecho de que el rediseño de la ciudad se encuentra normalizado por un positivismo intransigente, que incorpora a la vez que excluye a los especialistas del diseño de la ciudad y, por si no fuera poco, a la propia sociedad, por conducto del soslayo del cumplimiento de la legislación establecida en favorecimiento del capital y de prácticas administrativas de poca claridad, se contempla que si se logra establecer un diálogo Estado-SociedadEspecialistas del Diseño de la Ciudad. Si se da dicho diálogo sería posible concebir ciudades que faciliten que el ser humano disfrute de espacios físicos adaptados integrales que superen marginación y mínimos de bienestar. 


\section{Conclusiones}

En la medida que se establezcan diálogos como aquellos de los que se habla, entre Estado-Sociedad-Especialistas del Diseño de la Ciudad, es seguro que se encontrarán estructuras sólidas que implementen la normatividad y la legalidad por conducto de políticas públicas eficientes y eficaces; si bien es cierto que quizás con ello no se subsanarán las problemáticas a corto o mediano plazo, sí se sentarán las bases que permitan un rediseño de la ciudad en función de los cambios o exigencias de toda sociedad.

Además, se podrán incorporar elementos naturales inherentes al propio diseño de la ciudad (ciudades verdes, ciudades inteligentes, entre otras), en la implementación de mecanismos tecnológicos apropiados, que permitan establecer nuevos enfoques y replanteamientos de sus objetos de diseño y de sus métodos proyectuales, posibilitando la superación de viejos patrones y paradigmas, en aras de legitimar las acciones del Estado democrático - que en teoría debe prevalecer- en torno a la convivencia de sociedades urbanamente sanas. 


\section{Notas}

${ }^{1}$ Sin que con ello se pretenda decir que dichas problemáticas sean exclusivas de las mismas.

2 Disponible en Diario Oficial de la Federación en Línea: http://dof.gob.mx/nota detalle.php? codigo $=4845406 \&$ fecha $=26 / 05 / 1976$. Fecha de consulta: 21/07/2018.

3 Entendidos por la propia Ley General de Asentamientos Humanos, como la radicación de un determinado conglomerado demográfico con el conjunto de sus sistemas de convivencia, en un área físicamente localizada, considerando dentro de la misma los elementos naturales y las obras materiales que la integran.

${ }^{4}$ La cual se creó en la administración de José López Portillo. 


\section{Referencias}

Esquivel, N. (2012). Trazos para una ética hermenéutica en la vida y obra de Hans-Georg Gadamer. México: Torres Asociados.

Esquivel, N. (2016). Jürgen Habermas: acción comunicativa y ética del discurso. México: Gedisa.

Gobierno de México. (1976). Ley General de Asentamientos Humanos (LGAH). Diario Oficial de la Federación. Recuperado de http://dof.gob.mx/nota_ detalle.php?

Gutiérrez, J. J. (2009). Planeación urbana en México: un análisis crítico sobre su proceso de evolución. Revista Urbano, 12 (19), 52-63. Recuperado de http://www.redalyc.org/articulo.oa?id=19811644008

Kant, E. (2010). Critica de la razón pura. Madrid: Gredos.

Rousseau, J. (2011). Discurso sobre las ciencias y las artes. Madrid: Gredos.

Secretaría de Desarrollo Urbano y Metropolitano. (2018). Modificación al Plan Municipal de Desarrollo Urbano de Toluca 2018. Recuperado de http://seduv.edomexico.gob.mx/planes_municipales/toluca/PMD Utol.pdf 